Gynäkologische Endokrinologie 2011 . 9:141-142

DOI 10.1007/s10304-011-0436-8

Online publiziert: 2 . September 2011

(c) Springer-Verlag 2011

\author{
K. Diedrich \\ Klinik für Frauenheilkunde und Geburtshilfe, \\ Universitätsklinikum Schleswig-Holstein, Campus Lübeck
}

\title{
Zulässigkeit der Präimplantationsdiagnostik in Deutschland
}

beiden Partnern nach strengen Kriterien erfolgen. Zur Gewährleistung eines hohen medizinischen Standards soll die PID an lizenzierten Zentren vorgenommen werden. Die weiteren Durchführungsbestimmungen werden wahrscheinlich vom Wissenschaftlichen Beirat der Bundesärztekammer erarbeitet. Bereits 2003 wurde ein Diskussionsentwurf zu Richtlinien für die PID von der Bundesärztekammer vorgelegt. Dieser Diskussionsentwurf müsste jetzt entsprechend überarbeitet werden.

\section{(?) Jährlich werden nicht mehr als 200 PID-Fälle erwartet} grund dieses Urteils war der Gesetzgeber zum Handeln aufgefordert und kam nach langer Diskussion der rechtlichen und ethischen Gesichtspunkte im Bundestag schließlich zu einer Entscheidung. Es standen 3 Gesetzentwürfe zur Diskussion, wobei letztlich der Vorschlag von P. Hintze und U. Flach die Mehrheit erhielt. Danach soll die PID in Ausnahmefällen zulässig sein. Um Rechtssicherheit für Ärzte und die betroffenen Paare zu schaffen, ist das Embryonenschutzgesetz um eine Regelung zu ergänzen, welche die Voraussetzungen und das Verfahren einer PID festlegt. Zur Vermeidung eines Missbrauchs soll die PID nur nach verpflichtender Aufklärung und Beratung sowie dem positiven Votum einer interdisziplinär zusammengesetzten Ethikkommission zulässig sein. Des Weiteren ist sie nur in Fällen erlaubt, in denen einer oder beide Elternteile die Veranlagung für eine schwerwiegende Erbkrankheit tragen oder mit einer Totoder Fehlgeburt zu rechnen ist. Im Vorfeld der PID soll eine sorgfältige Diagnostik bei geben. Nicht mehr als 200 PID-Fälle werden erwartet.

Die Stellungnahme der Leopoldina hat in der Bundestagsdebatte eine wichtige Rolle gespielt. Auch eine Arbeitsgruppe des Wissenschaftlichen Beirats der Bundesärztekammer hat ein Memorandum zur PID erarbeitet. Sowohl in der Arbeitsgruppe der Leopoldina als auch im Wissenschaftlichen Beirat der Bundesärztekammer haben Experten der Deutschen Gesellschaft für Gynäkologie und Geburtshilfe (DGGG) intensiv mitgearbeitet. Das Memorandum der Bundesärztekammer wurde vom Vorstand verabschiedet. Der Antrag auf Zulassung der PID erhielt nach intensiver Debatte auf dem Deutschen Ärztetag in Kiel im Mai 2011204 Ja- und 33 Nein-Stimmen bei 6 Enthaltungen. Auf dem Deutschen Ärztetag 2003 in Rostock wurde die Zulassung noch mehrheitlich abgelehnt. Gemäß dem Memorandum soll die PID bei Erkrankungen durchgeführt werden, für die ein Paar ein hohes genetisches Risiko trägt. Die Bundesärztekammer wird sich dafür einsetzen, dass bei den Landesärztekammern PID-Kommissionen gebildet werden, die jeden Einzelfall anonym prüfen.

Bereits 1995 stellte die Universitätsfrauenklinik Lübeck gemeinsam mit Prof. E. Schwinger vom Institut für Humangenetik einen Antrag an die Ethikkommission der Universität, die Durchführung einer PID bei einem Paar zu erlauben, das durch eine Mukoviszidoseveranlagung belastet war. Es war bereits die Geburt eines Kindes mit Mukoviszidose vorausgegangen, das 2 Jahre nach der Geburt verstarb. Bei 2 nachfolgenden Schwangerschaften wurde nach pränataler Diagnostik ebenfalls die 
Mukoviszidoseveranlagung erkannt. Die Eltern entschieden sich nach entsprechender Beratung zu einem Schwangerschaftsabbruch. Die Ethikkommission der Universität Lübeck konnte zwar aus ethischen Gründen einer PID zustimmen, man sah jedoch einen Widerspruch zum bestehenden Embryonenschutzgesetz. Die Ethikkommission hat uns aufgefordert, für eine entsprechende Änderung des Embryonenschutzgesetzes Sorge zu tragen, welche die PID bei bestimmten Indikationsstellungen legalisieren würde.

Trotz langjähriger Diskussionen zwischen Vertretern der DGGG und der Politik, gemeinsam mit der Bundesärztekammer, konnte erst jetzt die gesetzliche Grundlage für die Zulässigkeit der PID geschaffen werden. Dies ist ein später, aber doch großer Fortschritt für die Gynäkologie in Deutschland. Damit werden endlich die Auslandsreisen unnötig, die Paare unternehmen, um eine PID durchführen zu lassen.

Nach dem Urteil des Bundesgerichtshofs zur Zulässigkeit der PID bei strenger Indikationsstellung haben wir in Lübeck mit der Anwendung begonnen. Im April konnte eine Schwangerschaft nach PID erreicht werden. In diesem Fall bestand eine Veranlagung für das DesbuquoisSyndrom, eine monogenetisch vererbte Skelettanomalie, bei der die Kinder meist intrauterin oder früh nach der Geburt sterben, da sich Lunge und Herz nicht entwickeln können. Das Paar hatte aufgrund dieses Syndroms bereits 3 Schwangerschaften mit intrauterinem Fruchttod hinter sich. Nach hormoneller Stimulation, In-vitroFertilisation der Eizellen und Biopsie von 2 Blastomeren im 10- bis 16-Zellstadium mit genetischer Untersuchung der Blastomeren konnten 2 genetisch unbelastete Embryonen transferiert werden; ein Embryo nistete sich in die Gebärmutterschleimhaut ein. Die Schwangerschaft verläuft problemlos und ist jetzt (3. August 2011) in der 14. Schwangerschaftswoche.

\section{Korrespondenzadresse}

Prof. Dr. Dr. h. c. mult. K. Diedrich

Klinik für Frauenheilkunde und

Geburtshilfe, Universitätsklinikum

Schleswig-Holstein, Campus Lübeck

Ratzeburger Allee 160, 23562 Lübeck

klaus.diedrich@uk-sh.de

\section{Themenübersicht}

Die „Gynäkologische Endokrinologie“ bietet Ihnen umfassende und aktuelle Beiträge zu interessanten Themenschwerpunkten aus allen Bereichen der gynäkologischen Endokrinologie und Reproduktionsmedizin.

Wir haben die Jahrgänge 2010/2011 im Überblick für Sie zusammengestellt:

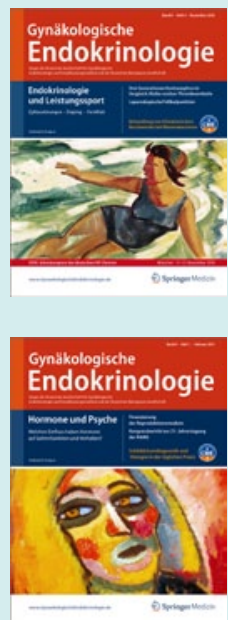

2010

- Heft 1/10 Onkologische Endokrinologie

- Heft 2/10 Mythen in der Reproduktionsmedizin

- Heft 3/10 Ovar: von der Fetalperiode bis zur Menopause

- Heft 4/10 Endokrinologie und Leistungssport

2011

- Heft 1/11 Hormone und Psyche

- Heft 2/11 Differenzialdiagnostik und Therapieoptionen der Hyperandrogenämie

- Heft 3/11 Menopause und Frauengesundheit

- Heft 4/11 Reproduktionsmedizin

Bestellen Sie einzelne Ausgaben oder abonnieren Sie die Zeitschrift zum Preis von EURO 125,- pro Jahr (zzgl. Versandkosten, Ermäßigung für Ärzte in Aus- und Weiterbildung und Fachgesellschaften)! Unser Kundenservice steht Ihnen für Fragen und Informationen gerne zur Verfügung:

Springer-Verlag, Kundenservice Zeitschriften

Haberstrasse 7, 69126 Heidelberg

Tel: + 496221/345-4303, Fax: +496221/345-4229

E-Mail: subscriptions@springer.com

\section{Beiträge auf einen Blick}

Abonennten haben online Zugriff auf alle Beiträge im elektronischen Volltextarchiv unter www.GynaekologischeEndokrinologie.de.

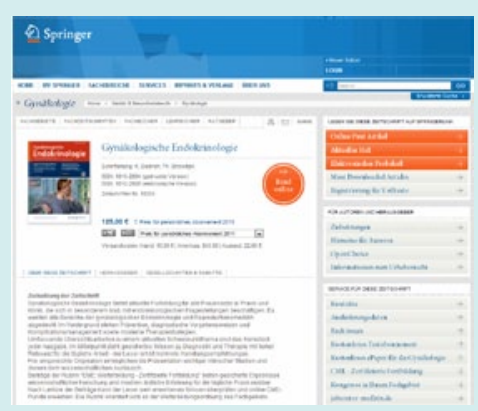

Eine interessante Lektüre wünscht Ihnen

Ihre Redaktion

Fachzeitschriften Medizin/ Psychologie
Ins Volltextarchiv gelangen Sie von der Startseite über den Navigationspunkt: "Read Online".

Nach einer einmaligen Registrierung können Sie die Beiträge als pdf oder in der html-Version abrufen. 